\title{
Comparing the Effects of Combined Oral Contraceptives Containing Progestins With Low Androgenic and Antiandrogenic Activities on the Hypothalamic-Pituitary-Gonadal Axis in Patients With Polycystic Ovary Syndrome: Systematic Review and Meta-Analysis
}

Mina Amiri ${ }^{1,2}, \mathrm{PhD}$, Postdoc; Fahimeh Ramezani Tehrani ${ }^{2}, \mathrm{MD}$; Fatemeh Nahidi ${ }^{3}, \mathrm{PhD}$; Ali Kabir ${ }^{4}, \mathrm{MD}, \mathrm{MPH}, \mathrm{PhD}$; Fereidoun $\mathrm{Azizi}^{5}$, MD

\footnotetext{
${ }^{1}$ Students Research Committee, School of Nursing and Midwifery, Department of Midwifery and Reproductive Health, Shahid Beheshti University of Medical Sciences, Tehran, Islamic Republic Of Iran

${ }^{2}$ Reproductive Endocrinology Research Center, Research Institute for Endocrine Sciences, Shahid Beheshti University of Medical Sciences, Tehran, Islamic Republic Of Iran

${ }^{3}$ School of Nursing and Midwifery, Department of Midwifery and Reproductive Health, Shahid Beheshti University of Medical Sciences, Tehran, Islamic Republic Of Iran

${ }^{4}$ Minimally Invasive Surgery Research Center, Iran University of Medical Sciences, Tehran, Islamic Republic Of Iran

${ }^{5}$ Endocrine Research Center, Shahid Beheshti University of Medical Sciences, Tehran, Islamic Republic Of Iran
}

\section{Corresponding Author:}

Fahimeh Ramezani Tehrani, MD

Reproductive Endocrinology Research Center

Research Institute for Endocrine Sciences

Shahid Beheshti University of Medical Sciences

24 Parvaneh

Yaman Street, Velenjak, PO Box 19395-4763

Tehran, 1985717413

Islamic Republic Of Iran

Phone: 982122432500

Email: ramezani@endocrine.ac.ir

\section{Abstract}

Background: Different products of combined oral contraceptives (COCs) can improve clinical and biochemical findings in patients with polycystic ovary syndrome (PCOS) through suppression of the hypothalamic-pituitary-gonadal (HPG) axis.

Objective: This systematic review and meta-analysis aimed to compare the effects of COCs containing progestins with low androgenic and antiandrogenic activities on the HPG axis in patients with PCOS.

Methods: We searched PubMed, Scopus, Google Scholar, ScienceDirect, and Web of Science databases (1980-2017) to identify randomized controlled trials or nonrandomized studies investigating the effect of COCs containing progestins with low androgenic and antiandrogenic activities, including the products containing desogestrel, cyproterone acetate, and drospirenone, on the HPG axis in patients with PCOS. In this meta-analysis, fixed and random effect models were used. Outcomes of interest were weighted mean differences (WMD) of hormonal parameters, including the follicle-stimulating hormone (FSH), luteinizing hormone (LH), LH-to-FSH ratio, estradiol, total testosterone, and sex hormone-binding globulin. Potential sources of heterogeneity were investigated using meta-regression and subgroup analyses. Subgroup analyses were performed based on the used progestin compound and treatment duration. We assessed quality of included studies and their risk of bias using Cochrane guidelines. Publication bias was assessed using Egger test and funnel plot.

Results: COC use was significantly associated with a decrease in gonadotropin levels, including FSH and LH. Use of products containing cyproterone acetate was associated with a decrease in FSH levels after 3 months (WMD $=-0.48 ; 95 \%$ CI -0.81 to -0.15 ), 6 months (WMD $=-2.33 ; 95 \% \mathrm{CI}-3.48$ to -1.18 ), and 12 months (WMD $=-4.70 ; 95 \% \mathrm{CI}-4.98$ to -4.42 ) and a decrease in LH levels after 3 months (WMD=-3.57; 95\% CI -5.14 to -1.99 ), 6 months (WMD $=-5.68 ; 95 \%$ CI -9.57 to -1.80 ), and 12 
months (WMD=-11.60; 95\% CI -17.60 to -5.60). Use of COCs containing drospirenone for 6 months decreased FSH $(\mathrm{WMD}=-0.93 ; 95 \% \mathrm{CI}-1.79$ to -0.08$)$ and $\mathrm{LH}(\mathrm{WMD}=-4.59 ; 95 \% \mathrm{CI}-7.53$ to -1.66$)$ levels. Data for products containing desogestrel were few, but this compound generally had no statistically significant influence on gonadotropin levels similar to that observed with COCs containing cyproterone acetate and drospirenone. Use of COCs was not associated with any significant change in LH-to-FSH ratio. COCs containing cyproterone acetate showed maximum effect on gonadotropin suppression. COCs containing cyproterone acetate significantly decreased estradiol concentrations, whereas those containing drospirenone exhibited no such effect. All COCs demonstrated improvement in androgenic profile and had the same effects on total testosterone and sex hormone-binding globulin concentrations. Progestin compound and treatment duration had no statistically significant effects on changing total testosterone and sex hormone-binding globulin levels.

Conclusions: COCs containing cyproterone acetate can effectively suppress gonadotropins, leading to a decrease in androgenic parameters. Although different products of COCs could significantly suppress the androgenic profile, it seems that products containing cyproterone acetate are more effective in suppressing gonadotropin and estradiol levels in patients with PCOS.

(JMIR Res Protoc 2018;7(4):e113) doi: 10.2196/resprot.9024

\section{KEYWORDS}

meta-analysis; combined oral contraceptives; androgens; gonadotropins; polycystic ovary syndrome

\section{Introduction}

Polycystic ovary syndrome (PCOS) is a common endocrine and metabolic disorder in reproductive age women [1-3], characterized by chronic oligo and/anovulation and hyperandrogenism (HA), which results in infertility, menstrual irregularities, hirsutism, acne, and alopecia [4]. PCOS is associated with an increase in risk of metabolic disorders such as obesity, dyslipidemia, and impaired glucose metabolism, which in turn increase the risk of diabetes mellitus and cardiovascular disease $[3,5,6]$. This endocrine disorder can have negative effects on the health-related quality of life of these women [7].

Combined oral contraceptives (COCs) are considered as the most common symptomatic treatment of PCOS and contain a combination of estrogen and progestin [8]. COCs are used not only to regulate menstrual cycle but also to suppress the hypothalamic-pituitary-gonadal (HPG) axis and improve clinical and biochemical HA in women with PCOS [9].

The effectiveness of COCs for the treatment of PCOS is well documented [10]. Previous studies show that COCs affect androgen synthesis by inhibiting ovarian androgen production [11-13]. The main potential mechanisms of COC action include inhibition of folliculogenesis as a result of suppression of gonadotropin secretion, suppression of ovarian and adrenal androgen synthesis, inhibition of 5 alpha reductase, and increased sex hormone-binding globulin (SHBG) [14,15]. Hence, COCs can improve the HPG axis function through a decrease in gonadotropin and ovarian androgen levels, which is a major goal of PCOS treatment [16].

Progestin activity of COCs inhibits luteinizing hormone (LH) secretion and results in a decline in ovarian androgen release [17]. Current COC products containing newer progestins with low androgenic or antiandrogenic effects, such as cyproterone acetate (CA), chlormadinone acetate (CMA), desogestrel (DSG), and drospirenone (DRSP), are considered to be effective in decreasing gonadotropin and androgen levels [14,18,19]. In particular, these progestins are better for women with PCOS suffering from HA [17].
Although the effect of COCs on the HPG axis of PCOS women has been introduced before, however, to the best of our knowledge, there is no other meta-analysis comparing this effect among COCs with various progesterone components. In our opinion, this is a valuable piece of knowledge that could provide some clues for a better understanding of the mechanism of effect of various COC compounds, which may be helpful in the decision-making process for treatment options.

This meta-analysis aimed to compare the effects of COCs containing progestins with low androgenic and antiandrogenic activities on the HPG axis in patients with PCOS.

\section{Methods}

\section{Overview}

This systematic review and meta-analysis was designed according to the PRISMA (Preferred Reporting Items for Systematic Reviews and Meta-Analyses) statement (Multimedia Appendix 1) [20] and the Cochrane Handbook for Systematic Reviews of Interventions [21] to answer the following questions:

1. Do COCs affect the HPG axis of women with PCOS?

2. Is there any difference among the effects of COCs on the HPG axis in women with PCOS?

3. Is there any difference in the effects of these compounds based on the duration of their use?

The study was approved by the ethics committee of Research Institute for Endocrine Sciences, Shahid Beheshti University of Medical Sciences, Tehran, Iran (Registration number: IR.SBMU.RIES.RES.1394.90).

\section{Search Strategy}

PubMed, Scopus, Google Scholar, ScienceDirect, and Web of Science were searched for clinical trials investigating the influence of COCs containing progestins with low androgenic and antiandrogenic activities on the HPG axis in patients with PCOS from January 1980 to June 2017. After searching for subheadings of PCOS in MeSH, the following keyword combinations were selected: ["polycystic ovary syndrome" AND "contracept"”] and ["polycystic ovary syndrome" AND 
"contraceptives, oral hormonal" OR "pill” OR "progestin"]. Search limitations were human, females, clinical trial, and English language.

A hand search of the reference lists of all selected papers was also conducted to prevent missing studies.

\section{Eligibility Criteria}

Studies conducted on reproductive age women with PCOS who were treated with monophasic COCs were selected for this meta-analysis; these studies were randomized clinical trials (RCTs) or nonrandomized studies (NRS).

Diagnostic criteria of each study are identified in Multimedia Appendix 2. In all the included studies, nonclassic congenital adrenal hyperplasia, hyperprolactynemia, and other HA etiologies were ruled out. The intervention of interest was COC containing progestins with low androgenic or antiandrogenic activities. Follicle-stimulating hormone (FSH), LH, LH-to-FSH ratio, estradiol (E2), total testosterone (TT), and SHBG levels were considered as main outcomes of the study.

Exclusion criteria were as follows: (1) women with idiopathic hirsutism or other types of HA, (2) women with diabetes or other chronic diseases, (3) use of biphasic or triphasic contraceptives, (4) use of gonadotropin-releasing hormone agonist-antagonist and antiandrogen drugs (eg, ketoconazole and spironolactone), (5) studies with follow-ups of $<3$ months or >24 months, (6) use of biphasic and triphasic COCs, (7) use of progesterone-only compounds, (8) use of metformin in combination with $\mathrm{COC}$, and (9) treatment groups with inadequate number of participants for performing meta-analysis (<1 study)

Only one study had a follow-up of 24 months and was excluded from the analysis [9]. We also excluded intervention groups that had no adequate number of study participants for performing a meta-analysis, including products containing levonorgestrel (LNG) and gestodene (GSD). In addition, study groups that assessed metformin + COCs were excluded from the analysis.

\section{Study Selection}

We included all relevant RCTs or NRS assessing COC effects on the HPG axis in reproductive age women with PCOS. At least one of the following hormonal parameters had to be reported: FSH, LH, LH-to-FSH ratio, E2, TT, or SHBG. We considered COCs containing CPA, DRSP, DSG, and CMA as interventions of interest. Due to inadequate number of studies that assessed products with LNG and DSG, these were excluded from the study.

The results of the searches were screened for meeting the predefined eligibility criteria. All references were entered to the endnote software. Selection was performed based on their titles, followed by using a second selection performed by 1 reviewer (MA), who deleted duplicates and reviewed abstracts of all remaining records. Any disagreement in the selection of abstracts was resolved by consensus or by another reviewer (FRT). Full-text articles for review and data processing were obtained for all selected abstracts.

\section{Data Extraction}

For each study, the following information were extracted: authors, year of publication, title, study design, characteristics of study population, type of intervention, outcome measurements-including FSH, milliunits per milliliters (mU/mL); LH, mU/mL; E2, picograms per milliliter; TT, nanograms per milliliter; and SHBG, nanomole per liter-and analytical methods. After data extraction, all the measurement units of hormones were identical. Data were extracted from full-text articles by 2 reviewers (MA and AK) in close consultation with another reviewer (FRT).

Data of studies were extracted by mean and SD [22]. To prevent extraction errors, a control check between the final data used in the meta-analysis and the original publications was performed by all authors.

\section{Quality Assessment}

Two reviewers (MA and AK) assessed the quality of the studies separately. They were blinded to study author, institution, and journal name. Disagreement was resolved and adjusted by the senior reviewer (FRT). A validated quality assessment checklist for clinical trial as the modified Consolidated Standards of Reporting Trials (CONSORT) was used to assign a score to each paper. The quality assessment of RCTs was assessed based on the 37-item CONSORT checklist. Each of the 37 items included in CONSORT were scored to compute an overall quality score (range 0-37). For scoring of the quality of items, 1 point was given if the information for each item was stated in the study, and 0 was given if the information was not stated or was unclear. CONSORT was also modified to the NRS, which were not randomized controlled studies. For modification of the checklist, questions related to the blinding and randomization were excluded.

All clinical trial papers were categorized into 4 groups: high, moderate, low, and very low quality. Studies with scores $\geq 70 \%$ of the highest level of the CONSORT checklist were considered as high, $40 \%$ to $70 \%$ as moderate, $20 \%$ to $40 \%$ as low, and $<20 \%$ as very low quality [23].

\section{Risk of Bias Assessment}

Two authors (MA and AK) independently assessed risk of bias. The risk of bias in each included study was assessed using the criteria outlined in the Cochrane Handbook for Systematic Reviews of Interventions [21,24,25]. Six domains related to risk of bias were assessed in each included RCTs: (1) random sequence generation; (2) allocation concealment; (3) blinding of participants and personnel; (4) blinding of outcome assessment; (5) incomplete outcome data; and (6) selective reporting. Review authors' judgments were categorized as "low risk," "high risk," and "unclear risk" of bias [24].

For NRS, 7 domains were assessed, including (1) confounding, (2) enrollment of participants in the study, (3) classification of interventions, (4) deviations from intended, (5) missing data, (6) measurement of outcomes, and (7) selection of the reported results. Review authors' judgments were categorized as "low risk," "moderate risk," "serious risk," "critical or high risk," and "unclear or no information risk" of bias [25]. 
We planned to assess outcomes based on the risk of bias in the following subgroups: (1) low risk, (2) moderate risk, (3) serious/high/critical risk, and (4) unclear or no information risk.

\section{Statistical Methods}

The studies selected assessed the effects of one or more COCs. Means and SDs of data at baseline and after treatment were collected. For studies reporting median and range, a conversion to mean and SD was performed, when possible [26]. Differences of mean and SD at both baseline and at end of treatment were calculated, as were standard errors of these differences, using the Cochrane Reviewers' Handbook. For effect measures, the mean difference [27] and related 95\% CIs were calculated based on the means of the pretreatment and those at the end of treatment levels of FSH, LH, LH-to-FSH ratio, E2, TT, and SHBG. Therefore, the primary pooled effect analysis was estimated weighted mean differences (WMD) for the studies comparing treatment groups of studies. For the end-of-treatment time point, the assessment (mean/SD) after a 3-, 6-, or 12-month cycle was used [21].

Heterogeneity tests were assessed by I-squared and chi-squared tests [28]. Both fixed and random effect models were used in the study. The random effect estimation method was applied for significant chi-squared test results $(P<.10)$ or I-squared greater than $50 \%$.

Subgroup analyses were performed based on COC compound and duration of use. In addition to funnel plot, Begg test [27] and Egger test [29] were used to assess publication bias. Publication bias was found to be significant for $P$ values <.10 to indicate significant asymmetry. For significant results or asymmetric funnel plot, the trim and fill method (by metatrim) was used to identify and correct for publication bias. Metatrim is a command used in the STATA version 12 software (StataCorp, College Station, TX, USA) for overcoming publication bias. It simulates studies that have not been published in literature and assesses whether the results would be different when there is or there is no publication bias. Indeed, for significant results or asymmetric funnel plot, the trim and fill method (by metatrim) was used to identify and correct for publication bias by adding some study measures [30,31]. We used meta-regression to evaluate heterogeneity induced by important variables, including diagnostic criteria of PCO (Rotterdam; National Institutes of Health, NIH; Androgen Excess Society, AES]; and others), body mass index (BMI), and method of assay of different hormones (radioimmunoassay, chemical/electrochemical luminescence, enzyme, and unknown). In addition, we used metainf for performing the sensitivity analysis. We also assessed risk of bias for included studies using the Risk of Bias tools as per the Cochrane guidelines, which are tools designed for RCTs and NRS [24,25]. We then performed a subgroup analysis based on the risk of bias. $P$ values <.05 were considered significant for all comparisons, except for heterogeneity, publication bias, and meta-regression, where .10 was set as the significance level. All analyses were performed with STATA software, version 12.

\section{Results}

\section{Search Results, Study Selection, Study Characteristics, and Quality Assessment}

A total of 1310 studies were retrieved by searching the electronic databases. After removing duplicates and assessing for quality appraisal and eligibility criteria, 34 studies were selected for the final analyses, which had 46 treatment groups (Figure 1 and Multimedia Appendix 2). Among these, 19 studies were RCTs and 15 studies were NRS. In all, 6 studies were classified as high, 20 as moderate, and 8 as low quality; 6 studies were identified as very low quality and were excluded from the meta-analysis. In most of the included studies $(n=25)$, PCOS was diagnosed by Rotterdam criteria. For other studies, NIH $(n=4)$ and AES criteria $(n=2)$ for diagnosing PCOS were used. Only 2 studies did not report their PCOS criteria. Also, for one study, we used the Homburg criteria for diagnosing PCOS. Ethinyl estradiol (EE) was the estrogenic component of COCs in all studies, whereas the progestin components were CA, DSG, DRSP, or CMA. Of 46 study arms, 20 were exposed to EE 35 $\mu \mathrm{g}+\mathrm{CA} 2 \mathrm{mg}[2,4,10,32-47], 17$ to EE $30 \mu \mathrm{g}+$ DRSP $3 \mathrm{mg}$ $[1,4,9,10,19,42,45,48-56], 6$ to EE $30 \mu \mathrm{g}+\mathrm{DSG} 150 \mu \mathrm{g}$ and 3 to EE $30 \mu \mathrm{g}+$ CMA $2 \mathrm{mg}$ (Multimedia Appendix 2) [9,53,57].

The study population consisted of 1224 women with PCOS with a mean age of 24.20 (95\% CI 23.19-25.30) years and a mean BMI of 24.42 (95\% CI 23.83-25.74) kg/m² (Multimedia Appendix 2). Sufficient data were collected for treatments of 3,6 , and 12 months but not for treatments of 24 months. All hormonal measurements of the studies were performed during the early follicular phase. Only 2 studies did not report days of hormonal assessment [22,58]. The effects of different COC treatments are summarized in Table 1 and Multimedia Appendices 3 and 4.

\section{Follicle-Stimulating Hormone}

A total of 13 studies reported effects of COCs on FSH. No study assessed the effects on FSH of EE + CMA for 3 to 12 months and EE + DSG for 12 months.

The use of EE + CA for 3 months was significantly associated with a decrease in FSH concentrations (WMD $=-0.48 ; 95 \% \mathrm{CI}$ -0.81 to -0.15 ), whereas use of EE + DSG or use of EE+ DRSP were not significantly associated. After 6 months of treatment with $\mathrm{EE}+\mathrm{CA}(\mathrm{WMD}=-2.33$; 95\% CI -3.48 to -1.18$)$ and DRSP (WMD $=-0.93 ; 95 \%$ CI -1.79 to -0.08$)$, FSH concentrations decreased, but there was no decrease with EE + DSG use. Use of EE + CA for 12 months was associated with a decrease in $\mathrm{FSH}$ concentrations (WMD $=-4.70$; 95\% CI -4.98 to -4.42), whereas the use of EE +DRSP was not. A significant heterogeneity was identified among most comparisons made with the FSH concentrations (Table 1 and Multimedia Appendices 3-5).

\section{Luteinizing Hormone}

A total of 18 studies reported the effects of COCs on LH. No study assessed the effects on LH of EE + CMA for 3 to 12 months and EE + DSG for 12 months. 
LH concentrations significantly decreased after 3 months of treatment with $\mathrm{EE}+\mathrm{DSG}(\mathrm{WMD}=-11.68 ; 95 \% \mathrm{CI}-13.72$ to -9.64) and $\mathrm{EE}+\mathrm{CA}(\mathrm{WMD}=-3.57$; $95 \% \mathrm{CI}-5.14$ to -1.99$)$ but not with EE + DRSP. After 6 months of treatment with EE $+\mathrm{CA}(\mathrm{WMD}=-5.68 ; 95 \% \mathrm{CI}-9.57$ to -1.80$)$ and $\mathrm{EE}+\mathrm{DRSP}$ $(\mathrm{WMD}=-4.59 ; 95 \% \mathrm{CI}-7.53$ to -1.66$)$, LH concentrations significantly decreased, whereas no significant decrease in concentration was observed with EE + DSG use (Figure 2). The use of $\mathrm{EE}+\mathrm{CA}(\mathrm{WMD}=-11.60 ; 95 \% \mathrm{CI}-17.60$ to -5.60$)$ for 12 months also decreased LH concentrations, whereas use of $\mathrm{EE}+\mathrm{DRSP}$ did not. There was significant heterogeneity among some comparisons (Table 1 and Multimedia Appendices 3-5).

Figure 1. Flow diagram of literature search and study selection. PCOS: polycystic ovary syndrome; GnRH: gonadotropin-releasing hormone; COC: combined oral contraceptives.
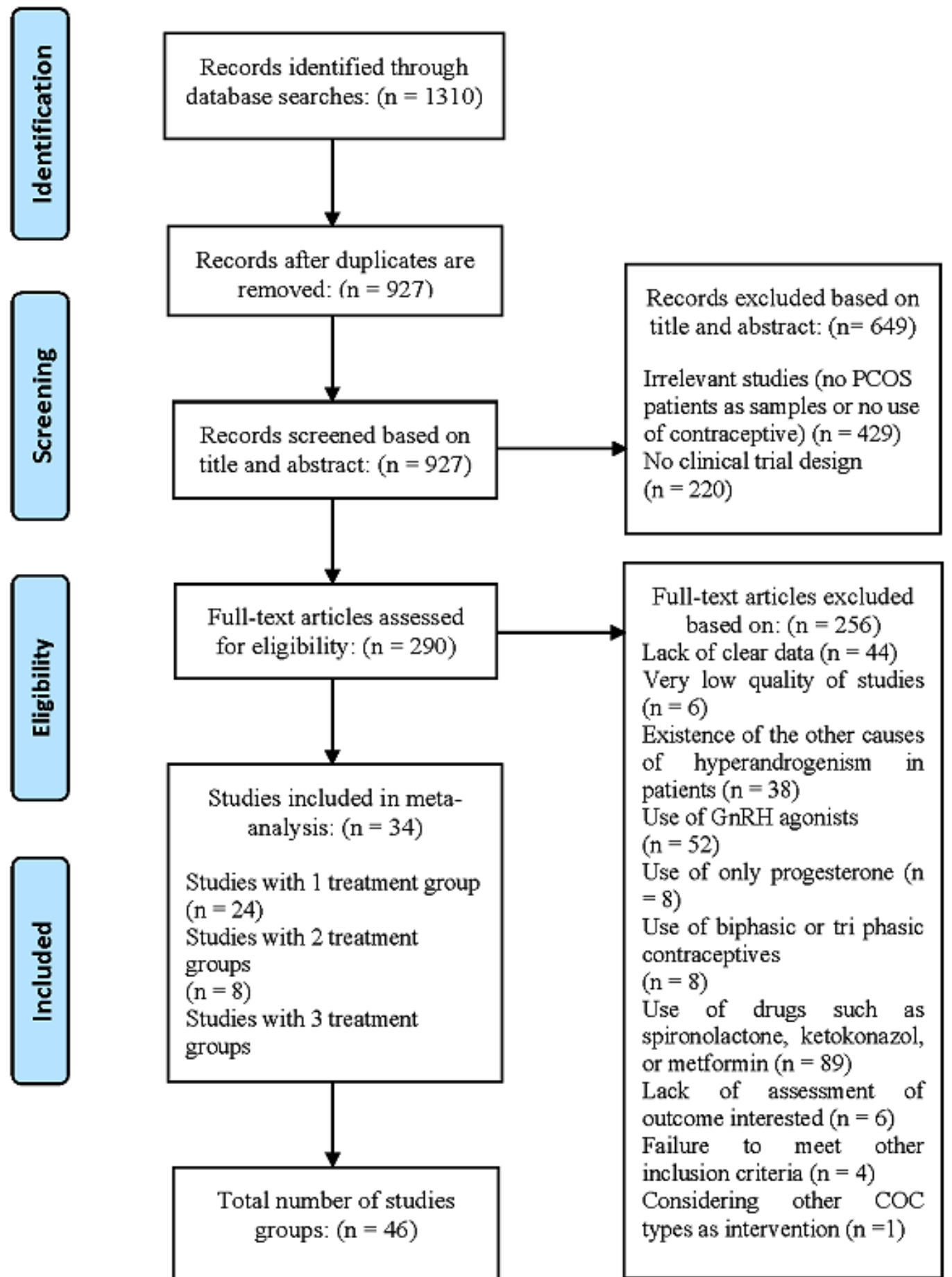
Table 1. Effects of different combined oral contraceptives on hormonal parameters in women with polycystic ovary syndrome. $\uparrow$ and $\downarrow$ indicate increase and decrease, respectively.

\begin{tabular}{|c|c|c|c|c|}
\hline Hormonal parameters & $\mathrm{EE}^{\mathrm{a}}+\mathrm{CA}^{\mathrm{b}}$ & $\mathrm{EE}+\mathrm{DRSP}^{\mathrm{c}}$ & $\mathrm{EE}+\mathrm{CMA}^{\mathrm{d}}$ & $\mathrm{EE}+\mathrm{DSG}^{\mathrm{e}}$ \\
\hline \multicolumn{5}{|l|}{$\mathbf{F S H}^{\mathbf{f}}$} \\
\hline 3 months & $\downarrow$ & $\mathrm{NO}^{\mathrm{g}}$ & N/A ${ }^{h}$ & NO \\
\hline 6 months & $\downarrow$ & $\downarrow$ & N/A & NO \\
\hline 12 months & $\downarrow$ & NO & N/A & N/A \\
\hline \multicolumn{5}{|l|}{$\mathbf{L} \mathbf{H}^{\mathrm{i}}$} \\
\hline 3 months & $\downarrow$ & NO & N/A & $\downarrow$ \\
\hline 6 months & $\downarrow$ & $\downarrow$ & N/A & NO \\
\hline 12 months & $\downarrow$ & NO & N/A & N/A \\
\hline \multicolumn{5}{|l|}{ LH-to-FSH ratio } \\
\hline 3 months & NO & NO & N/A & NO \\
\hline 6 months & NO & NO & N/A & NO \\
\hline 12 months & NO & NO & N/A & N/A \\
\hline \multicolumn{5}{|l|}{$\mathbf{E} 2^{\mathbf{j}}$} \\
\hline 3 months & $\downarrow$ & NO & N/A & N/A \\
\hline 6 months & $\downarrow$ & NO & N/A & N/A \\
\hline 12 months & $\downarrow$ & NO & N/A & N/A \\
\hline \multicolumn{5}{|l|}{$\mathbf{T T}^{\mathbf{k}}$} \\
\hline 3 months & $\downarrow$ & $\downarrow$ & $\downarrow$ & $\downarrow$ \\
\hline 6 months & $\downarrow$ & $\downarrow$ & $\downarrow$ & $\downarrow$ \\
\hline 12 months & $\downarrow$ & $\downarrow$ & $\downarrow$ & NO \\
\hline \multicolumn{5}{|l|}{ SHBG $^{1}$} \\
\hline 3 months & $\uparrow$ & $\uparrow$ & $\uparrow$ & $\uparrow$ \\
\hline 6 months & $\uparrow$ & $\uparrow$ & NO & $\uparrow$ \\
\hline 12 months & $\uparrow$ & $\uparrow$ & $\uparrow$ & $\uparrow$ \\
\hline
\end{tabular}

${ }^{\mathrm{a} E E}$ : ethinyl estradiol.

${ }^{\mathrm{b}} \mathrm{CA}$ : cyproterone acetate.

${ }^{\mathrm{c}}$ DRSP: drospirenone.

${ }^{\mathrm{d}} \mathrm{CMA}$ : chlormadinone acetate.

${ }^{\mathrm{e} D S G}$ : desogestrel.

${ }^{f}$ FSH: follicle-stimulating hormone.

${ }^{\mathrm{g}} \mathrm{NO}$ : no significant effect.

${ }^{\mathrm{h}} \mathrm{N} / \mathrm{A}$ : not assessed.

${ }^{\mathrm{i}} \mathrm{LH}$ : luteinizing hormone.

${ }^{\mathrm{j}} \mathrm{E} 2$ : estradiol.

${ }^{\mathrm{k}} \mathrm{TT}$ : total testosterone.

${ }^{1}$ SHBG: sex hormone-binding globulin. 
Figure 2. Forest plot of combined oral contraceptives' effects on luteinizing hormone after 6 months of treatment.

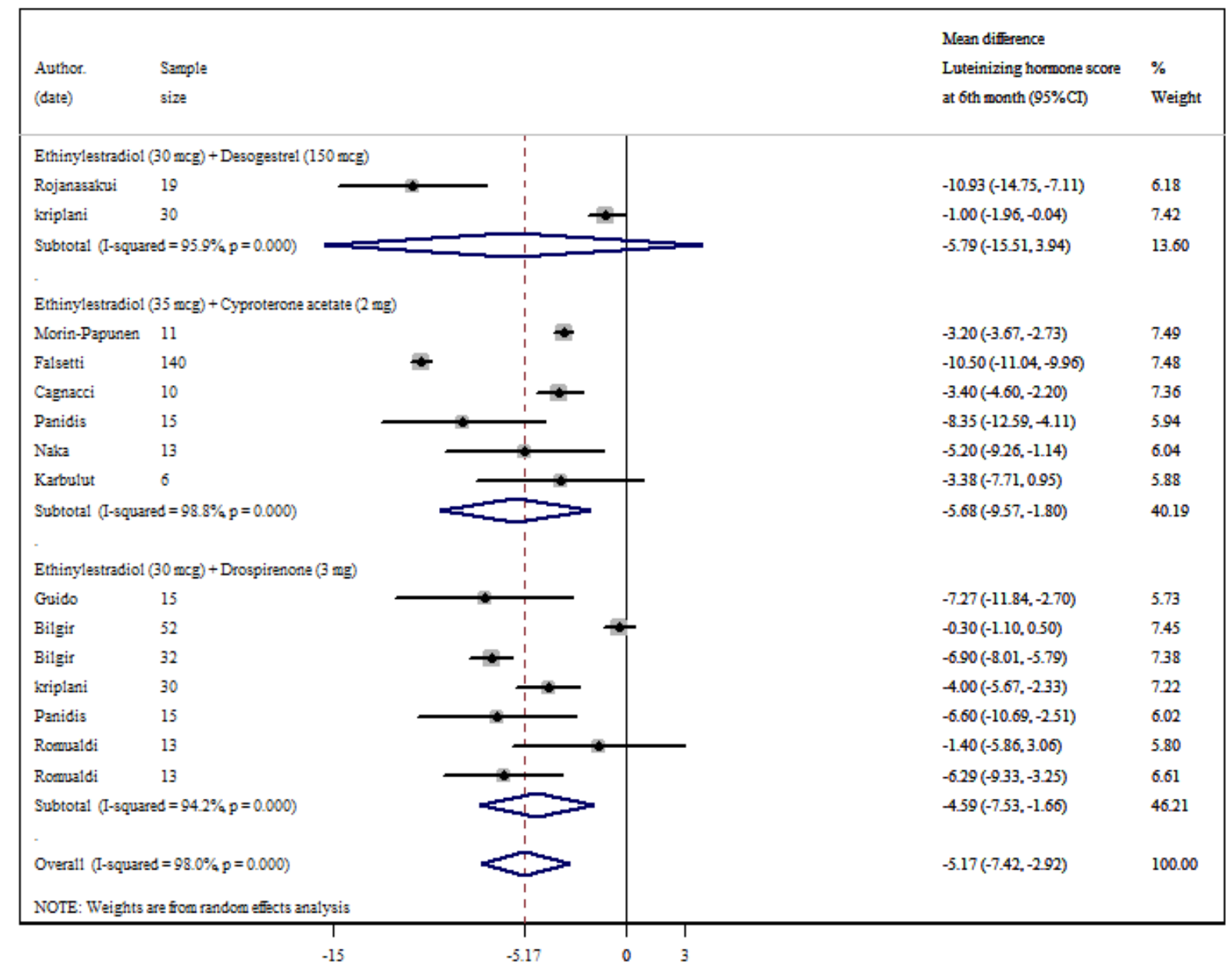

\section{Luteinizing Hormone to Follicle-Stimulating Hormone Ratio}

A total of 13 studies reported the effects of COCs on LH-to-FSH ratio. No study assessed the effect on LH-to-FSH ratio of EE + CMA for 3 to 12 months and EE + DSG for 12 months.

The use of $\mathrm{EE}+\mathrm{DSG}, \mathrm{EE}+\mathrm{CA}$, and $\mathrm{EE}+\mathrm{CMA}$ for 3 to 12 months was not associated with any significant change in LH-to-FSH ratio (Table 1 and Multimedia Appendices 3-5).

\section{Estradiol}

A total of 7 studies reported the effects of COCs on E2, whereas no study assessed the effect on $\mathrm{E} 2$ of EE + CMA and EE + DSG for 3 to 12 months.

The use of $\mathrm{EE}+\mathrm{CA}$ for 3 to 12 months significantly decreased the $\mathrm{E} 2$ concentrations; WMDs $(95 \% \mathrm{CI})$ in these durations of follow-ups were $-5.62(-10.49$ to -0.75$),-28.90(-31.44$ to $-26.36)$, and -32.43 ( -46.11 to -18.74$)$, respectively. EE + DRSP use was not associated with any significant change in E2 concentrations. No significant heterogeneity was identified among comparisons (Table 1 and Multimedia Appendices 3-5).

\section{Total Testosterone}

A total of 30 studies reported the effects of COCs on TT.
The use of various COCs, including EE + DSG (WMD $=-0.41$; $95 \% \mathrm{CI}-0.73$ to -0.08$), \mathrm{EE}+\mathrm{CA}(\mathrm{WMD}=-0.25 ; 95 \% \mathrm{CI}-0.29$ to -0.21 ), and $\mathrm{EE}+\mathrm{DRSP}(\mathrm{WMD}=-0.22 ; 95 \% \mathrm{CI}-0.38$ to $-0.05)$, was associated with a significant decrease in TT after 3 months of treatment; however, there was no decrease after $\mathrm{EE}+\mathrm{CMA}$ use. After 6 months of use, all treatments including $\mathrm{EE}+\mathrm{DSG}(\mathrm{WMD}=-0.20 ; 95 \% \mathrm{CI}-0.36$ to -0.04$), \mathrm{EE}+\mathrm{CA}$ $(\mathrm{WMD}=-0.30 ; 95 \% \mathrm{CI}-0.44$ to -0.16$), \mathrm{EE}+\mathrm{DRSP}$ $(\mathrm{WMD}=-0.17 ; 95 \% \mathrm{CI}-0.23$ to -0.11$)$, and $\mathrm{EE}+\mathrm{CMA}$ (WMD $=-0.24 ; 95 \%$ CI -0.37 to -0.11 ) decreased TT concentrations. The 12-month use of $\mathrm{EE}+\mathrm{CA}(\mathrm{WMD}=-0.29$; $95 \% \mathrm{CI}-0.54$ to -0.04$), \mathrm{EE}+\mathrm{DRSP}(\mathrm{WMD}=-0.12 ; 95 \% \mathrm{CI}$ -0.22 to -0.03 ), and $\mathrm{EE}+\mathrm{CMA}(\mathrm{WMD}=-0.10 ; 95 \% \mathrm{CI}-0.17$ to -0.03 ) also decreased TT concentrations, although EE + DSG use was not associated with any significant change in TT. For all comparisons made with TT concentrations, significant heterogeneity was identified (Table 1 and Multimedia Appendices 3-5).

\section{Sex Hormone-Binding Globulin}

A total of 25 studies reported the effects of COCs on SHBG.

Different COCs containing EE + DSG (WMD=99; 95\% CI 88.74-109.26), $\mathrm{EE}+\mathrm{CA}(\mathrm{WMD}=96.86$; $95 \%$ CI 47.88-145.84), $\mathrm{EE}+\mathrm{DRSP}(\mathrm{WMD}=100.90 ; 95 \%$ CI 12.50-189.30), and $\mathrm{EE}+$ CMA (WMD=137.73; 95\% CI 89.14-186.32) were associated with increase in SHBG concentrations, following 3 months of 
treatment. After 6 months of treatment, $\mathrm{EE}+\mathrm{DSG}$ $(\mathrm{WMD}=57.35 ; 95 \%$ CI 19.59-95.11), $\mathrm{EE}+\mathrm{CA}(\mathrm{WMD}=102.17$; 95\% CI 82.72-121.63), EE + DRSP (WMD=93.54; 95\% CI 63.63-123.45) increased SHBG concentrations, whereas EE + CMA use was not associated with any significant change in SHBG. SHBG concentrations were also increased after 12 months of treatment with all COCs, including $\mathrm{EE}+\mathrm{DSG}$ $(\mathrm{WMD}=181.98 ; 95 \%$ CI 20.25-343.71), $\mathrm{EE}+\mathrm{CA}$ $(\mathrm{WMD}=162.10 ; 95 \%$ CI 101.63-222.56), $\mathrm{EE}+\mathrm{DRSP}$ $(\mathrm{WMD}=89.33 ; 95 \%$ CI 41.45-137.21), and $\mathrm{EE}+\mathrm{CMA}$ (WMD=9.24; 95\% CI 6.65-11.83). There was significant heterogeneity identified among comparisons (Table 1 and Multimedia Appendices 3-5).

\section{Publication Bias}

The results of Egger test showed a significant publication bias for FSH $(P=.01)$ and E2 after 6 months $(P=.07)$, and corrections were performed on the outcomes. Metatrim showed a change (from mean difference, $\mathrm{MD}=-1.30 ; 95 \% \mathrm{CI}-2.14$ to -0.46 to $\mathrm{MD}=-1.33 ; 95 \% \mathrm{CI}-2.16$ to -0.49$)$ for FSH after 6 months but no change for $\mathrm{E} 2(\mathrm{MD}=-8.96$; $95 \% \mathrm{CI}-24.16$ to 6.24$)$ in women with PCOS after correcting for publication bias (Multimedia Appendix 6). Other publication biases were not significant.

\section{Meta-Regression Analysis}

We used meta-regression to evaluate heterogeneity induced by variables, including diagnostic criteria of PCOS (Rotterdam, NIH, AES, and other), BMI, and method of assay of different hormones (radioimmunoassay, chemical/electrochemical luminescence, enzyme, and unknown). Our univariate meta-regression analysis showed that BMI has a significant effect on FSH difference at 6th month compared with baseline level (beta=.55; $P=.096$ ). Diagnostic criteria of PCOS were also a significant source of heterogeneity for FSH difference at the 6th month from the baseline level $(P=.02)$ and SHBG difference at the 12th month from the baseline level (beta=-4.21; $P=.002$ ). The method of assay was also a significant source of heterogeneity for TT difference at the 6th month from the baseline level (beta $=-.29 ; P=.007$ ) and SHBG difference at the 6 th month from the baseline level (beta $=44.45 ; P=.085$ ). None of the potential confounders had any effect on LH and E2 levels. As previously mentioned, for meta-regression, a $P$ value $<.10$ was considered statistically significant.

Our multivariate meta-regression was done only for FSH difference at the 6th month from the baseline level, which had more than one source of heterogeneity: BMI and diagnostic criteria of PCOS. It showed that only diagnostic criteria of PCOS is a significant source of heterogeneity (beta=-4.46; $P=.059$ ). We did not use multivariate meta-regression for other variables because none of them had more than 1 source for their heterogeneity among the 3 variables, including BMI, diagnostic criteria of PCOS, and method of assay.

\section{Sensitivity Analysis}

The results of metainf showed that there are few studies that can distort the results. Most of the time the point estimates and 95\% CIs are in a specified similar limit with others, which showed homogeneity among the studies. We can hence ignore the risk of introducing bias by BMI, diagnostic criteria of PCOS, or method of assay. Details of the sensitivity analysis are presented in Multimedia Appendix 7.

\section{Risk of Bias Assessment}

Multimedia Appendices 8 and 9 show details of risk of bias of published studies. Most RCT studies were at low risk of bias of random sequence generation $(52 \%, 10 / 19)$, blinding of participants and personnel $(63 \%, 17 / 19)$, and selective outcome reporting $(89 \%, 17 / 19$; in these studies, some biases were more probable such as blinding of outcome assessment and incomplete outcome data (Multimedia Appendix 8). The NRS were not at a high risk of bias. . They had a low risk bias for classification of interventions and selection of reported results (Multimedia Appendix 9).

Generally, most studies had an acceptable validity (low risk of bias), demonstrating high quality of these studies in most aspects. Subgroup analysis based on the risk of bias showed no significant change in outcomes, indicating logical generalizability of these studies.

\section{Discussion}

\section{Principal Findings}

This meta-analysis compared the effects of COCs with progestins containing low androgenic and antiandrogenic activities on the HPG axis in patients with PCOS. A total of 34 studies involving 1224 women was included in this analysis. Findings showed that the use of COCs containing CA was significantly associated with a decrease in gonadotropins (FSH and LH) and E2 concentrations, whereas COCs containing DRSP did not change these parameters. Data were insufficient to assess the effects of COCs containing CMA and DSG on gonadotropins and E2, but in general these products had no significant effects on these hormonal parameters. COCs were not associated with any significant change in the LH-to-FSH ratio. The use of all COCs was associated with an increase in SHBG and decrease in TT levels, except for DSG at 12 month and CMA at 6 month of treatment.

PCOS has a complex pathogenesis and is believed to be a result of disturbances in gonadotropin secretion. Abnormal secretion of gonadotropins, particularly LH, from the pituitary gland leads to abnormal and excessive ovarian theca cell androgens [59].

Estrogen and progestin components of COCs act together to suppress FSH and LH secretion and the midcycle gonadotropin surge by a feedback mechanism, which results in a decrease in ovarian steroidogenesis $[49,60,61]$. Indeed, suppression of LH is the major mechanism that mediates the effects of these products in PCOS patients [62].

This study showed that COC use was significantly associated with a suppression of gonadotropin (FSH and $\mathrm{LH}$ ) levels. Duration of treatment is considered to be an important factor in the suppression of gonadotropins. In fact, the use of products containing CA for 3 to 12 months was associated with a decrease in FSH and LH levels, whereas COCs containing DRSP decreased these hormones only after 6 months of treatment. Thus, products containing DRSP generally require a more 
prolonged usage to suppress the gonadotropins. Data for products containing DSG are limited, but this compound generally had no influence on gonadotropin levels similar to that observed with COCs containing CA and DRSP. COCs containing $\mathrm{CA}$ are associated with higher gonadotropin suppression compared with that of other COCs.

No studies assessed the effect of EE + DSG, EE + CMA, and $\mathrm{EE}+\mathrm{GSD}$ on E2 levels. Therefore, data were available only for evaluating the effect of compounds containing EE $35 \mu \mathrm{g}+$ CA $2 \mathrm{mg}$ and EE $30 \mu \mathrm{g}+$ DRSP $3 \mathrm{mg}$ on E2 levels. This analysis demonstrated that COCs containing CA significantly decreased E2 concentrations, whereas COCs containing DRSP exhibited no such effect. Duration of treatment with COCs was not significant on E2 concentrations. This review clearly shows that COCs containing CA are more effective compared with COCs containing DRSP on E2 levels; however, these data are not sufficient to assess the effect of other contraceptives on E2.

Testosterone, a major androgen in women, increases in many PCOS patients. Although all COCs can decrease androgen levels by gonadotropin suppression, contraceptives with antiandrogen progestins have additional specific mechanisms in addition to the main mechanisms to improve HA $[16,63]$. Therefore, it can be suggested that gonadotropins are independent of sex steroid secretion [64]. Similar to all progestins, antiandrogen progestins inhibit $\mathrm{LH}$ and increase clearance of testosterone, which leads to a decrease in androgen levels $[65,66]$. These newer progestins also exert antiandrogenic effects by competing at the receptor sites with androgens and inhibit 5 alpha-reductase activity $[16,65]$. Five alpha reductase enzyme catalyzes testosterone to dihydrotestosterone [67]. This key enzyme is necessary for biosynthesis and metabolism of androgens [68]. Adipose tissue is an important source of active steroid production and metabolism. It contains the aromatase enzyme that converts circulating androgens to estrogens. As some estrogens in premenopausal women originate from the peripheral conversion of androgens, the plasma concentrations of estrone and E2 may be significantly correlated with the extent of adipose mass. Obesity is associated with several abnormalities in androgen metabolism [69]. Urinary excretion of SHBG is lower in obese women compared with normal-weight women [70]. Kirschner et al found that menopausal women with abdominal obesity had higher testosterone levels than those with peripheral obesity [71]. Obesity is associated with increased androgen production rate and metabolic clearance rate; however, the main differences are higher estrogen and lower SHBG levels, whereas usually no differences are found in androgen and gonadotropin concentrations [69].

Interestingly, this study showed that all COCs containing CA, DRSP, CMA, and DSG can decrease TT concentrations. The type of COC and duration of treatment with COCs had no significant effects on TT concentrations. A meta-analysis assessed the effect of COCs on testosterone concentrations in healthy women and found that the progestin type of COCs does not affect the testosterone levels [66]; their findings are consistent with those of this meta-analysis. However, they did not evaluate the pituitary hormones and other hormones secreted by the ovary in women with PCOS.
This review also strongly demonstrates that SHBG significantly increased during the use of COCs. Progestin compound and duration of treatment had no important effects on the changes in SHBG levels.

Comparative studies are not adequate to assess the effect of COCs on HPG; therefore, this meta-analysis included NRS and individual arms of RCTs. However, it is well known that meta-analysis of NRS can produce equally or more precise findings for a clinical question compared with meta-analysis of RCTs alone [3,72].

\section{Limitations}

This study has several limitations that should be mentioned. First, there is no single definition for the diagnosis of PCOS and its components. Second, different studies assessed hormonal measurements by different methods. Third, some studies did not assess all hormonal parameters. Finally, some subgroups in this meta-analysis had limited studies to analyze, which can affect the robustness of the results. Therefore, additional studies are required to provide more concrete data to investigate and confirm the accuracy of this conclusion.

There was significant heterogeneity in most outcomes, which can reflect clinical heterogeneity related to variability in PCOS diagnostic criteria; interpretation of laboratory tests; study population, for example, age; BMI; ethnicity or race; and methods used to measure hormones. To deal with significant heterogeneities, we used the random effect model. A majority of studies included in this analysis used the Rotterdam criteria for diagnosing PCOS and a limited number used other diagnostic criteria, such as AES or NIH. Therefore, this meta-analysis has also included PCOS patients with oligomenorrea and cystic ovaries without clinical or biochemical HA. The results of this study showed that diagnostic criteria of PCOS can also be a source of heterogeneity for FSH concentrations, indicating that variability in diagnostic criteria can be a cause of differences in gonadotropin concentrations. However, a sensitivity analysis showed that most of the time point estimates and 95\% CIs are in a specified limit similar to those of others; hence, we can ignore the risk of introducing bias by BMI, diagnostic criteria of PCOS, or method of assay. Some included studies reported the early follicular phase as the timing of hormonal measurement without determining its exact time; however, blood samples for all studies were collected at early follicular phase of the spontaneous menstrual cycle or progesterone-induced menstrual bleeding.

To minimize selection bias, study selection was conducted based on the eligibility criteria, which had been accurately determined just before the study. A hand search of the reference lists of all selected papers was also conducted to prevent missing studies.

The pooled estimate of this meta-analysis provides precise results as it has acceptable risk of bias and publication bias; in addition, it included studies conducted among reproductive age women from various regions of the world. Moreover, a subgroup analysis based on the risk of bias was not associated with any significant differences in outcomes. Hence, we can rely on the pooled estimate and the generalizability of these studies. 
In this review, the difference between baseline and posttreatment levels was calculated to assess the effects of COCs on the HPG axis. However, subgroup analyses based on different baseline levels and PCOS phenotypes were not performed because of the limitations of the existing studies.

\section{Conclusions}

This meta-analysis indicates that COC use for 3 to 12 months can suppress gonadotropins, leading to a decrease in androgenic profiles in women with PCOS. Although progestin compounds used and duration of treatment were not effective in reducing the circulating levels of androgens and SHBG, they were important in gonadotropin suppression. This study demonstrates that products containing CA have the greatest suppressive effect on gonadotropins and E2, indicating that the use of this compound may be a better alternative for PCOS patients with impaired gonadotropins. However, because of the limitations of the data available for comparison of the effects of all COCs on HPG, the investigators recommend designing further comparative studies.

\section{Acknowledgments}

The authors thank Mrs N Shiva for critical editing of English grammar and syntax of the manuscript. The authors also thank the Institute of Endocrine Sciences of Shahid Beheshti University of Medical Sciences for approval of this project and its funding as the research project.

\section{Authors' Contributions}

MA was involved in the study design, search in databases, study selection, data analysis, manuscript drafting, and critical discussion. FRT was involved in the study design, data analysis, critical discussion, and editing and submission of the manuscript. AK contributed to the study selection, data analysis, and critical discussion. FN and FA contributed to the study design and critical discussion. All authors have read and approved the final manuscript.

\section{Conflicts of Interest}

None declared.

\section{Multimedia Appendix 1}

PRISMA (Preferred Reporting Items checklist for Systematic Reviews and Meta-Analyses) 2009 checklist.

[PDF File (Adobe PDF File), 60KB-Multimedia Appendix 1]

\section{Multimedia Appendix 2}

Characteristics of eligible studies included in the meta-analysis.

[PDF File (Adobe PDF File), 55KB-Multimedia Appendix 2]

\section{Multimedia Appendix 3}

Effects of EE $35+$ CA 2 and EE $30+$ DRSP 3 on hormonal parameters.

[PDF File (Adobe PDF File), 33KB-Multimedia Appendix 3]

\section{Multimedia Appendix 4}

Effects of EE 30 + CMA 2 and EE 30 + DSG 150 on hormonal parameters.

[PDF File (Adobe PDF File), 32KB-Multimedia Appendix 4]

\section{Multimedia Appendix 5}

Forest plots of combined oral contraceptives' effects on hormonal parameters, including the follicle-stimulating hormone, luteinizing hormone, estradiol, total testosterone, and sex hormone-binding globulin.

[PDF File (Adobe PDF File), 119KB-Multimedia Appendix 5]

\section{Multimedia Appendix 6}

Funnel plots of publication bias and related corrections.

[PDF File (Adobe PDF File), 151KB-Multimedia Appendix 6] 


\section{Multimedia Appendix 7}

The results of sensitivity analysis.

[PDF File (Adobe PDF File), 86KB-Multimedia Appendix 7]

\section{Multimedia Appendix 8}

Risk of bias for randomized controlled trials.

[JPG File, 105KB-Multimedia Appendix 8]

\section{Multimedia Appendix 9}

Risk of bias for nonrandomized studies.

[JPG File, 90KB-Multimedia Appendix 9]

\section{References}

1. Kriplani A, Periyasamy AJ, Agarwal N, Kulshrestha V, Kumar A, Ammini AC. Effect of oral contraceptive containing ethinyl estradiol combined with drospirenone vs. desogestrel on clinical and biochemical parameters in patients with polycystic ovary syndrome. Contraception 2010 Aug;82(2):139-146. [doi: 10.1016/j.contraception.2010.02.009] [Medline: 20654754]

2. Elter K, Imir G, Durmusoglu F. Clinical, endocrine and metabolic effects of metformin added to ethinyl estradiol-cyproterone acetate in non-obese women with polycystic ovarian syndrome: a randomized controlled study. Hum Reprod 2002 Jul;17(7):1729-1737. [Medline: 12093831]

3. Amiri M, Ramezani Tehrani F, Nahidi F, Kabir A, Azizi F, Carmina E. Effects of oral contraceptives on metabolic profile in women with polycystic ovary syndrome: a meta-analysis comparing products containing cyproterone acetate with third generation progestins. Metabolism 2017 Aug;73:22-35. [doi: 10.1016/j.metabol.2017.05.001] [Medline: 28732568]

4. Kahraman K, Sükür YE, Atabekoğlu CS, Ateş C, Taşkın S, Cetinkaya SE, et al. Comparison of two oral contraceptive forms containing cyproterone acetate and drospirenone in the treatment of patients with polycystic ovary syndrome: a randomized clinical trial. Arch Gynecol Obstet 2014 Aug;290(2):321-328. [doi: 10.1007/s00404-014-3217-5] [Medline: 24676694]

5. Robinson S, Henderson AD, Gelding SV, Kiddy D, Niththyananthan R, Bush A, et al. Dyslipidaemia is associated with insulin resistance in women with polycystic ovaries. Clin Endocrinol (Oxf) 1996 Mar;44(3):277-284. [Medline: 8729522]

6. Amiri M, Mirmiran P, Ramezani Tehrani P, Ramezani Tehrani F. Effect of interventions based on lifestyle modification on clinical, hormonal and metabolic findings in the patients with polycystic ovary syndrome: a systematic review. Iran $\mathbf{J}$ Endocrinol Metab 2016 Mar 16;17(6):489-500 [FREE Full text]

7. Hahn S, Janssen OE, Tan S, Pleger K, Mann K, Schedlowski M, et al. Clinical and psychological correlates of quality-of-life in polycystic ovary syndrome. Eur J Endocrinol 2005 Dec;153(6):853-860 [FREE Full text] [doi: 10.1530/eje.1.02024] [Medline: 16322391]

8. Yildiz BO. Approach to the patient: contraception in women with polycystic ovary syndrome. J Clin Endocrinol Metab 2015 Mar;100(3):794-802. [doi: 10.1210/jc.2014-3196] [Medline: 25701301]

9. Yildizhan R, Gokce AI, Yildizhan B, Cim N. Comparison of the effects of chlormadinone acetate versus drospirenone containing oral contraceptives on metabolic and hormonal parameters in women with PCOS for a period of two-year follow-up. Gynecol Endocrinol 2015 May;31(5):396-400. [doi: 10.3109/09513590.2015.1006187] [Medline: 25739031]

10. Bhattacharya SM, Jha A. Comparative study of the therapeutic effects of oral contraceptive pills containing desogestrel, cyproterone acetate, and drospirenone in patients with polycystic ovary syndrome. Fertil Steril 2012 Oct;98(4):1053-1059. [doi: 10.1016/j.fertnstert.2012.06.035] [Medline: 22795636]

11. Helvaci N, Yildiz BO. Oral contraceptives in polycystic ovary syndrome. Minerva Endocrinol 2014 Sep;39(3):175-187. [Medline: 25003228]

12. Breitkopf DM, Rosen MP, Young SL, Nagamani M. Efficacy of second versus third generation oral contraceptives in the treatment of hirsutism. Contraception 2003 May;67(5):349-353. [Medline: 12742556]

13. Rosen MP, Breitkopf DM, Nagamani M. A randomized controlled trial of second- versus third-generation oral contraceptives in the treatment of acne vulgaris. Am J Obstet Gynecol 2003 May;188(5):1158-1160. [Medline: 12748463]

14. Burkman Jr RT. The role of oral contraceptives in the treatment of hyperandrogenic disorders. Am J Med 1995 Jan 16;98(1A):130S-136S. [Medline: 7825633]

15. Raudrant D, Rabe T. Progestogens with antiandrogenic properties. Drugs 2003;63(5):463-492. [Medline: 12600226]

16. Mathur R, Levin O, Azziz R. Use of ethinylestradiol/drospirenone combination in patients with the polycystic ovary syndrome. Ther Clin Risk Manag 2008 Apr;4(2):487-492 [FREE Full text] [Medline: 18728832] 
17. Di Carlo C, Gargano V, Sparice S, Tommaselli GA, Bifulco G, Nappi C. Effects of an oral contraceptive containing estradiol valerate and dienogest on circulating androgen levels and acne in young patients with PCOS: an observational preliminary study. Gynecol Endocrinol 2013 Dec;29(12):1048-1050. [doi: 10.3109/09513590.2013.831834] [Medline: 24020909]

18. Sitruk-Ware R. Pharmacological profile of progestins. Maturitas 2008;61(1-2):151-157. [Medline: 19434887]

19. Palep-Singh M, Mook K, Barth J, Balen A. An observational study of Yasmin in the management of women with polycystic ovary syndrome. J Fam Plann Reprod Health Care 2004 Jul;30(3):163-165 [FREE Full text] [Medline: 15222920]

20. Moher D, Liberati A, Tetzlaff J, Altman DG, PRISMA Group. Preferred reporting items for systematic reviews and meta-analyses: the PRISMA statement. Int J Surg 2010;8(5):336-341 [FREE Full text] [doi: 10.1016/j.ijsu.2010.02.007] [Medline: 20171303]

21. Higgins JP, Green S. Cochrane Handbook for Systematic Reviews of Interventions, Vol 5. UK: Wiley Online Library; 2008.

22. Rojanasakul A, Sirimongkolkasem R, Piromsawasdi S, Sumavong V, Chailurkit L, Chaturachinda K. Effects of combined ethinylestradiol and desogestrel on hormone profiles and sex hormone binding globulin in women with polycystic ovarian disease. Contraception 1987 Dec;36(6):633-640. [Medline: 2965636]

23. Moher D, Hopewell S, Schulz KF, Montori V, Gøtzsche PC, Devereaux PJ, et al. CONSORT. Amsterdam, Netherlands: Elsevier; 2010:e1-e37.

24. Higgins JP, Altman DG, Gøtzsche PC, Jüni P, Moher D, Oxman AD, Cochrane Bias Methods Group, Cochrane Statistical Methods Group. The Cochrane Collaboration's tool for assessing risk of bias in randomised trials. Br Med J 2011;343:d5928 [FREE Full text] [Medline: 22008217]

25. Sterne JA, Hernán MA, Reeves BC, Savović J, Berkman ND, Viswanathan M, et al. ROBINS-I: a tool for assessing risk of bias in non-randomised studies of interventions. Br Med J 2016 Oct 12;355:i4919 [FREE Full text] [Medline: 27733354]

26. Hozo SP, Djulbegovic B, Hozo I. Estimating the mean and variance from the median, range, and the size of a sample. BMC Med Res Methodol 2005 Apr 20;5(1):13. [doi: 10.1186/1471-2288-5-13]

27. Begg CB, Mazumdar M. Operating characteristics of a rank correlation test for publication bias. Biometrics 1994 Dec;50(4):1088-1101. [Medline: 7786990]

28. Higgins JP, Thompson SG, Deeks JJ, Altman DG. Measuring inconsistency in meta-analyses. Br Med J 2003 Sep 6;327(7414):557-560 [FREE Full text] [doi: 10.1136/bmj.327.7414.557] [Medline: 12958120]

29. Egger M, Davey Smith G, Schneider M, Minder C. Bias in meta-analysis detected by a simple, graphical test. Br Med J 1997 Sep 13;315(7109):629-634 [FREE Full text] [Medline: 9310563]

30. Taylor S, Tweedie R. Trim and fill: a simple funnel plot based method of testing and adjusting for publication bias in meta-analyses. Fort Collins, CO: Colorado State University 1998:455-463.

31. Duval S, Tweedie R. Trim and fill: a simple funnel-plot-based method of testing and adjusting for publication bias in meta-analysis. Biometrics 2000 Jun;56(2):455-463. [Medline: 10877304]

32. Morin-Papunen LC, Vauhkonen I, Koivunen RM, Ruokonen A, Martikainen HK, Tapanainen JS. Endocrine and metabolic effects of metformin versus ethinyl estradiol-cyproterone acetate in obese women with polycystic ovary syndrome: a randomized study. J Clin Endocrinol Metab 2000 Sep;85(9):3161-3168. [doi: 10.1210/jcem.85.9.6792] [Medline: 10999803]

33. Armstrong VL, Wiggam MI, Ennis CN, Sheridan B, Traub AI, Atkinson AB, et al. Insulin action and insulin secretion in polycystic ovary syndrome treated with ethinyl oestradiol/cyproterone acetate. QJM 2001 Jan;94(1):31-37. [Medline: 11161134]

34. Falsetti L, Gambera A, Tisi G. Efficacy of the combination ethinyl oestradiol and cyproterone acetate on endocrine, clinical and ultrasonographic profile in polycystic ovarian syndrome. Hum Reprod 2001 Jan;16(1):36-42. [Medline: 11139533]

35. Mastorakos G, Koliopoulos C, Creatsas G. Androgen and lipid profiles in adolescents with polycystic ovary syndrome who were treated with two forms of combined oral contraceptives. Fertil Steril 2002 May;77(5):919-927. [Medline: 12009344]

36. Cagnacci A, Paoletti AM, Renzi A, Orrù M, Pilloni M, Melis GB, et al. Glucose metabolism and insulin resistance in women with polycystic ovary syndrome during therapy with oral contraceptives containing cyproterone acetate or desogestrel. J Clin Endocrinol Metab 2003 Aug;88(8):3621-3625. [doi: 10.1210/jc.2003-030328] [Medline: 12915645]

37. Lv L, Liu Y, Sun Y, Tan K. Effects of metformin combined with cyproterone acetate on clinical features, endocrine and metabolism of non-obese women with polycystic ovarian syndrome. J Huazhong Univ Sci Technolog Med Sci 2005;25(2):194-197. [Medline: 16116971]

38. Wu J, Zhu Y, Jiang Y, Cao Y. Effects of metformin and ethinyl estradiol-cyproterone acetate on clinical, endocrine and metabolic factors in women with polycystic ovary syndrome. Gynecol Endocrinol 2008 Jul;24(7):392-398. [doi: 10.1080/09513590802217027] [Medline: 18608522]

39. Gul OB, Somunkiran A, Yucel O, Demirci F, Ozdemir I. The effect of ethinyl estradiol-cyproterone acetate treatment on homocysteine levels in women with polycystic ovary syndrome. Arch Gynecol Obstet 2008 Jan;277(1):25-30. [doi: 10.1007/s00404-007-0401-x] [Medline: 17618446]

40. Bilgir O, Kebapcilar L, Taner C, Bilgir F, Kebapcilar A, Bozkaya G, et al. The effect of ethinylestradiol (EE)/cyproterone acetate (CA) and EE/CA plus metformin treatment on adhesion molecules in cases with polycystic ovary syndrome (PCOS). Intern Med 2009;48(14):1193-1199 [FREE Full text] [Medline: 19602786 ] 
41. Kebapcilar L, Yuksel A, Bozkaya G, Taner CE, Kebapcilar AG, Bilgir O, et al. Effects of an EE/CA compared with EE/CA-metformin on serum ADMA levels in women with polycystic ovary syndrome. Cent Eur J Med 2009 Dec;4(4):423-427 [FREE Full text] [doi: 10.2478/s11536-009-0074-x]

42. Mahmood M, El-Kattan E, El-Aal H, El LA, Ghamry N, Sheta M. Evaluation of the clinical and biochemical effects of medical therapy in women with polycystic ovary syndrome. Med J Cairo Univ 2009 Sep;77(2):59-67.

43. Chen MJ, Yang WS, Chen HF, Kuo JJ, Ho HN, Yang YS, et al. Increased follistatin levels after oral contraceptive treatment in obese and non-obese women with polycystic ovary syndrome. Hum Reprod 2010 Mar;25(3):779-785. [doi: 10.1093/humrep/dep459] [Medline: 20093255]

44. Teede HJ, Meyer C, Hutchison SK, Zoungas S, McGrath BP, Moran LJ. Endothelial function and insulin resistance in polycystic ovary syndrome: the effects of medical therapy. Fertil Steril 2010 Jan;93(1):184-191. [doi: 10.1016/j.fertnstert.2008.09.034] [Medline: 19019358]

45. Panidis D, Georgopoulos NA, Piouka A, Katsikis I, Saltamavros AD, Decavalas G, et al. The impact of oral contraceptives and metformin on anti-Müllerian hormone serum levels in women with polycystic ovary syndrome and biochemical hyperandrogenemia. Gynecol Endocrinol 2011 Aug;27(8):587-592. [doi: 10.3109/09513590.2010.507283] [Medline: $\underline{20836726}$ ]

46. Naka KK, Kalantaridou SN, Bechlioulis A, Kravariti M, Kazakos N, Katsouras CS, et al. Effect of ethinylestradiol/cyproterone acetate on endothelial function in young non-obese women with polycystic ovary syndrome: a pilot study. Gynecol Endocrinol 2011 Sep;27(9):615-621. [doi: 10.3109/09513590.2010.521265] [Medline: 21329419]

47. Karabulut A, Demirlenk S, Sevket O. Effects of ethinyl estradiol-cyproterone acetate treatment on metabolic syndrome, fat distribution and carotid intima media thickness in polycystic ovary syndrome. Gynecol Endocrinol 2012 Apr;28(4):245-248. [doi: 10.3109/09513590.2011.613971] [Medline: 21961995]

48. Guido M, Romualdi D, Giuliani M, Suriano R, Selvaggi L, Apa R, et al. Drospirenone for the treatment of hirsute women with polycystic ovary syndrome: a clinical, endocrinological, metabolic pilot study. J Clin Endocrinol Metab 2004 Jun;89(6):2817-2823. [doi: 10.1210/jc.2003-031158] [Medline: 15181063 ]

49. Pehlivanov B, Mitkov M. Efficacy of an oral contraceptive containing drospirenone in the treatment of women with polycystic ovary syndrome. Eur J Contracept Reprod Health Care 2007 Mar;12(1):30-35. [doi: 10.1080/13625180600983082] [Medline: 17455042]

50. Gregoriou O, Papadias K, Konidaris S, Bakalianou K, Salakos N, Vrachnis N, et al. Treatment of hirsutism with combined pill containing drospirenone. Gynecol Endocrinol 2008 Apr;24(4):220-223. [doi: 10.1080/09513590801948309] [Medline: 18382909]

51. Ozdemir S, Görkemli H, Gezginç K, Ozdemir M, Kiyici A. Clinical and metabolic effects of medroxyprogesterone acetate and ethinyl estradiol plus drospirenone in women with polycystic ovary syndrome. Int J Gynaecol Obstet 2008 Oct;103(1):44-49. [doi: 10.1016/j.ijgo.2008.05.017] [Medline: 18635183 ]

52. Sağsöz N, Orbak Z, Noyan V, Yücel A, Uçar B, Yildiz L. The effects of oral contraceptives including low-dose estrogen and drospirenone on the concentration of leptin and ghrelin in polycystic ovary syndrome. Fertil Steril 2009 Aug;92(2):660-666. [doi: 10.1016/j.fertnstert.2008.07.008] [Medline: 18973889]

53. Colonna L, Pacifico V, Lello S, Sorge R, Raskovic D, Primavera G. Skin improvement with two different oestroprogestins in patients affected by acne and polycystic ovary syndrome: clinical and instrumental evaluation. J Eur Acad Dermatol Venereol 2012 Nov;26(11):1364-1371. [doi: 10.1111/j.1468-3083.2011.04292.x] [Medline: 22011217]

54. Romualdi D, De Cicco S, Busacca M, Gagliano D, Lanzone A, Guido M. Clinical efficacy and metabolic impact of two different dosages of ethinyl-estradiol in association with drospirenone in normal-weight women with polycystic ovary syndrome: a randomized study. J Endocrinol Invest 2013 Sep;36(8):636-641. [doi: 10.1007/BF03346756] [Medline: 24105072]

55. Aydin K, Cinar N, Aksoy DY, Bozdag G, Yildiz BO. Body composition in lean women with polycystic ovary syndrome: effect of ethinyl estradiol and drospirenone combination. Contraception 2013 Mar;87(3):358-362. [doi: 10.1016/j.contraception.2012.07.005] [Medline: 22898361]

56. Macut D, Božić Antić I, Nestorov J, Topalović V, Bjekić Macut J, Panidis D, et al. The influence of combined oral contraceptives containing drospirenone on hypothalamic-pituitary-adrenocortical axis activity and glucocorticoid receptor expression and function in women with polycystic ovary syndrome. Hormones (Athens) 2015;14(1):109-117 [FREE Full text] [doi: 10.14310/horm.2002.1526] [Medline: 25402380]

57. De Leo V, Di Sabatino A, Musacchio MC, Morgante G, Scolaro V, Cianci A, et al. Effect of oral contraceptives on markers of hyperandrogenism and SHBG in women with polycystic ovary syndrome. Contraception 2010 Sep;82(3):276-280. [doi: 10.1016/j.contraception.2010.04.002] [Medline: 20705157]

58. Nader S, Riad-Gabriel MG, Saad MF. The effect of a desogestrel-containing oral contraceptive on glucose tolerance and leptin concentrations in hyperandrogenic women. J Clin Endocrinol Metab 1997 Sep;82(9):3074-3077. [doi: 10.1210/jcem.82.9.4192] [Medline: $\underline{9284746]}$

59. Ehrmann DA. Polycystic ovary syndrome. N Engl J Med 2005 Mar 24;352(12):1223-1236. [doi: 10.1056/NEJMra041536] [Medline: 15788499] 
60. Vrbíková J, Cibula D. Combined oral contraceptives in the treatment of polycystic ovary syndrome. Hum Reprod Update 2005;11(3):277-291. [doi: 10.1093/humupd/dmi005] [Medline: 15790599]

61. Uras R, Orrù M, Pani F, Marotto M, Pilloni M, Guerriero S, et al. Endocrinological, metabolic and clinical features of treatment with oral contraceptive formulation containing ethinylestradiol plus chlormadinone acetate in nonobese women with polycystic ovary syndrome. Contraception 2010 Aug;82(2):131-138. [doi: 10.1016/j.contraception.2010.01.022] [Medline: 20654753]

62. Carlström K, Karlsson R, Von Schoultz B. Diurnal rhythm and effects of oral contraceptives on serum dehydroepiandrosterone sulfate (DHEAS) are related to alterations in serum albumin rather than to changes in adrenocortical steroid secretion. Scand J Clin Lab Invest 2002;62(5):361-368. [Medline: 12387582]

63. Nader S, Diamanti-Kandarakis E. Polycystic ovary syndrome, oral contraceptives and metabolic issues: new perspectives and a unifying hypothesis. Hum Reprod 2007 Feb;22(2):317-322. [doi: 10.1093/humrep/del407] [Medline: 17099212]

64. Holte J, Bergh T, Gennarelli G, Wide L. The independent effects of polycystic ovary syndrome and obesity on serum concentrations of gonadotrophins and sex steroids in premenopausal women. Clin Endocrinol (Oxf) 1994 Oct;41(4):473-481. [Medline: 7955458]

65. Badawy A, Elnashar A. Treatment options for polycystic ovary syndrome. Int J Womens Health 2011 Feb 08;3:25-35 [FREE Full text] [doi: 10.2147/IJWH.S11304] [Medline: 21339935]

66. Zimmerman Y, Eijkemans MJ, Coelingh Bennink HJ, Blankenstein MA, Fauser BC. The effect of combined oral contraception on testosterone levels in healthy women: a systematic review and meta-analysis. Hum Reprod Update 2014;20(1):76-105 [FREE Full text] [doi: 10.1093/humupd/dmt038] [Medline: 24082040]

67. Longcope C. Adrenal and gonadal androgen secretion in normal females. Clin Endocrinol Metab 1986 May;15(2):213-228. [Medline: $\underline{3013468]}$

68. Thiboutot D, Jabara S, McAllister JM, Sivarajah A, Gilliland K, Cong Z, et al. Human skin is a steroidogenic tissue: steroidogenic enzymes and cofactors are expressed in epidermis, normal sebocytes, and an immortalized sebocyte cell line (SEB-1). J Invest Dermatol 2003 Jun;120(6):905-914 [FREE Full text] [doi: 10.1046/j.1523-1747.2003.12244.x] [Medline: 12787114]

69. Pasquali R, Casimirri F. The impact of obesity on hyperandrogenism and polycystic ovary syndrome in premenopausal women. Clin Endocrinol (Oxf) 1993 Jul;39(1):1-16. [Medline: 8348699]

70. Glass AR, Burman KD, Dahms WT, Boehm TM. Endocrine function in human obesity. Metabolism 1981 Jan;30(1):89-104. [Medline: 6780754]

71. Kirschner MA, Samojlik E, Drejka M, Szmal E, Schneider G, Ertel N. Androgen-estrogen metabolism in women with upper body versus lower body obesity. J Clin Endocrinol Metab 1990 Feb;70(2):473-479. [doi: 10.1210/jcem-70-2-473] [Medline: 2298859]

72. Shrier I, Boivin J, Steele RJ, Platt RW, Furlan A, Kakuma R, et al. Should meta-analyses of interventions include observational studies in addition to randomized controlled trials? A critical examination of underlying principles. Am J Epidemiol 2007 Nov 15;166(10):1203-1209. [doi: 10.1093/aje/kwm189] [Medline: 17712019]

\section{Abbreviations}

AES: Androgen Excess Society

BMI: body mass index

CA: cyproterone acetate

CMA: chlormadinone acetate

COCs: combined oral contraceptives

CONSORT: Consolidated Standards of Reporting Trials

DRSP: drospirenone

DSG: desogestrel

EE: ethinyl estradiol

E2: estradiol

FSH: follicle-stimulating hormone

GSD: gestodene

HA: hyperandrogenism

HPG: hypothalamic-pituitary-gonadal

LH: luteinizing hormone

LNG: levonorgestrel

MD: mean difference

NIH: National Institutes of Health

NO: no significant effect

NRS: nonrandomized studies

N/A: not assessed 
PCOS: polycystic ovary syndrome

PRISMA: Preferred Reporting Items checklist for Systematic Reviews and Meta-Analyses

RCT: randomized controlled trial

SHBG: sex hormone-binding globulin

TT: total testosterone

WMD: weighted mean differences

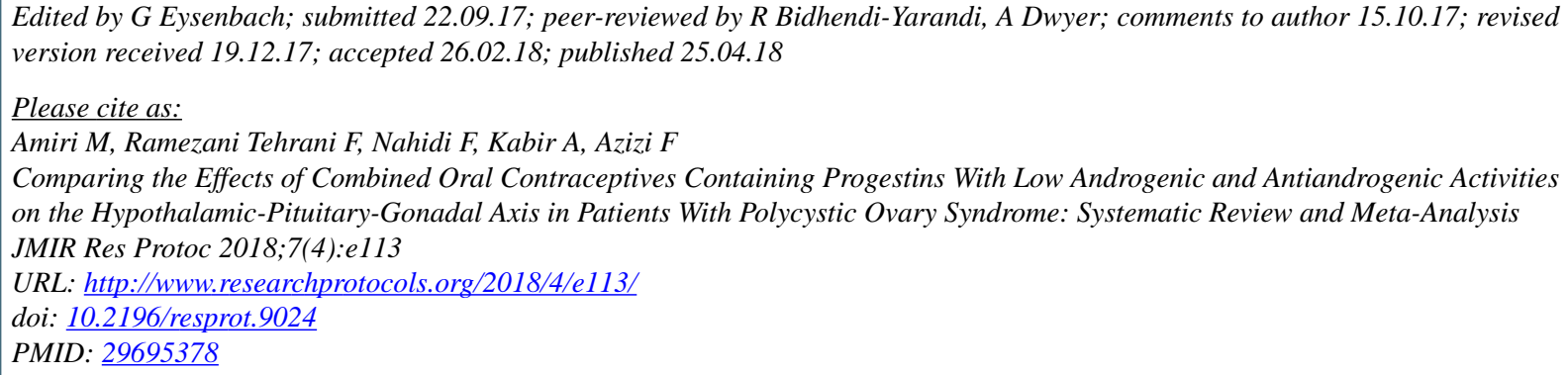

(CMina Amiri, Fahimeh Ramezani Tehrani, Fatemeh Nahidi, Ali Kabir, Fereidoun Azizi. Originally published in JMIR Research Protocols (http://www.researchprotocols.org), 25.04.2018. This is an open-access article distributed under the terms of the Creative Commons Attribution License (https://creativecommons.org/licenses/by/4.0/), which permits unrestricted use, distribution, and reproduction in any medium, provided the original work, first published in JMIR Research Protocols, is properly cited. The complete bibliographic information, a link to the original publication on http://www.researchprotocols.org, as well as this copyright and license information must be included. 